Check for updates

Cite this: RSC Adv., 2019, 9, 37171

Received 1st September 2019

Accepted 3rd November 2019

DOI: 10.1039/c9ra06934h

rsc.li/rsc-advances

\section{N, O and P co-doped honeycomb-like hierarchical porous carbon derived from Sophora japonica for high performance supercapacitors $\uparrow$}

\begin{abstract}
Li Zhang, * Yuxia Zhu, Guangzhen Zhao, (D) Yanjiang Li and Guang Zhu (D) *
Novel N, O and P co-doped honeycomb-like hierarchically porous carbon (N-O-P-HHPC) materials with a large specific surface area from Sophora japonica were prepared via a one-step activation and carbonization method and used as an electrode for supercapacitors. The results indicate that asprepared N-P-HHPC with a large specific surface area $\left(2068.9 \mathrm{~m}^{2} \mathrm{~g}^{-1}\right.$ ) and N (1.5 atomic\%), O (8.4 atomic\%) and $\mathrm{P}$ (0.4 atomic\%) co-doping has a high specific capacitance of $386 \mathrm{~F} \mathrm{~g}^{-1}$ at $1 \mathrm{~A} \mathrm{~g}^{-1}$. Moreover, a $1.8 \mathrm{~V}$ symmetrical SC was assembled from the $\mathrm{N}-\mathrm{O}-\mathrm{P}-\mathrm{HHPC}-3$ electrode using $1 \mathrm{M} \mathrm{Na}_{2} \mathrm{SO}_{4}$ gel electrolyte, presenting a high energy density $\left(28.4 \mathrm{~W} \mathrm{~h} \mathrm{~kg}^{-1}\right.$ at $\left.449.9 \mathrm{~W} \mathrm{~kg}^{-1}\right)$ and a long life cycling stability with only $7.3 \%$ capacitance loss after 10000 cycles. Furthermore, the coin-type symmetrical SC using EMIMBF4 as electrolyte presents an ultrahigh energy density $\left(80.8 \mathrm{~W} \mathrm{~h} \mathrm{~kg}^{-1}\right.$ at $\left.1500 \mathrm{~W} \mathrm{~kg}^{-1}\right)$. When the two coin-type symmetrical SCs are connected in series, eight red light-emitting diodes (LEDs) and a small display screen can be powered. These results demonstrate as-prepared N, O and P co-doped HHPC is a considerable candidate as a carbon electrode for energy storage devices.
\end{abstract}

\section{Introduction}

Along with the fast development of the global economy, the energy crisis and environmental pollution caused by the large consumption of non-renewable resources such as coal and oil, have become the main obstacles of economic development. ${ }^{1-3}$ Meanwhile, the development of electron devices and electric vehicles is an indispensable part of economic development. Therefore, developing efficient, green and stable energy storage devices is an important way to solve economic and environmental issues. ${ }^{4-8}$ Supercapacitors (SCs), also named as electrochemical capacitors, have received interest for portable and miniaturized electronics, electric vehicles, etc., due to their ultra-high power density, light weight, fast charge/discharge, outstanding stability and safety and so on. ${ }^{9,10}$ The electrode material as one of the most essential parts affects the electrochemical properties of SCs.

As we know, the charge storage of the carbon derived electrode material for SCs is that charge gathered on the surface to adsorb electrolyte ions. ${ }^{11-16}$ Over a long time, there are some strategies to enhance the electrochemical properties of carbon material, such as porous structure optimization, heteroatom doping and so on. ${ }^{17-20}$ It has been found that a reasonable interconnected

Key Laboratory of Spin Electron and Nanomaterials of Anhui Higher Education Institutes, Suzhou University, Suzhou 234000, PR China. E-mail: zhlisuzh@163.com; guangzhu@ahszu.edu.cn

$\dagger$ Electronic supplementary information (ESI) available. See DOI: 10.1039/c9ra06934h porous structure with micropores, mesopores and macropores, which can short the transmission path of ion electrolyte and provide enough adsorption sites for ion electrolyte, and thus can enhance the electrochemical performance. ${ }^{21,22}$ Therefore, carbon material with hierarchical porous nanostructure has became the focus of scholars' attention. On the other hand, the heteroatom $\left(\mathrm{N},{ }^{23} \mathrm{P}^{24} \mathrm{~S}^{25} \mathrm{~B}^{26}\right.$ and $\left.\mathrm{O}^{21}\right)$ doped into carbon material is an effective strategy to improve performance. It has been proved that the heteroatom doping can enhance the conductivity and wettability, and produce pseudocapacitance. Meanwhile, the multi-heteroatoms doping can further improve the capacitance, compared to the one-type-only heteroatom doping..$^{22,27}$

Biomass derived hierarchical porous carbon materials have aroused attention of scholars, owning to their advantages of environmental friendship, low cost and easy access. ${ }^{28,29}$ The most importantly, biomass based hierarchical porous carbon material can inherit nature unique microstructures and the chemical compositions, which can easily produce porous structure and multi-heteroatoms doping. ${ }^{30,31}$ To date, many hierarchical porous framework structures or multi-heteroatoms co-doped carbon materials have been prepared from biomass, such as laozao, ${ }^{32}$ soybeans, ${ }^{33}$ Perilla frutescens, ${ }^{34}$ rice husk. ${ }^{35}$ During the preparation process, $\mathrm{KOH}$ as a activating agent is widely used to etch the carbon matrix leaving porous framework structures (microporous and mesoporous) to create very high surface area. ${ }^{12,36}$ For instance, the specific capacitance of porous carbon derived from fungus with $\mathrm{KOH}$ chemical activation is $374 \mathrm{~F} \mathrm{~g}^{-1}$ at current density of $0.5 \mathrm{~A} \mathrm{~g}^{-1}$ than primeval carbon $\left(116 \mathrm{~F} \mathrm{~g}^{-1}\right){ }^{37}$ Biomass waste based activated carbon material 
displays a higher specific capacitance $\left(222 \mathrm{~F} \mathrm{~g}^{-1}\right.$ at $\left.1 \mathrm{~A} \mathrm{~g}^{-1}\right) .^{38}$ Recently, $\mathrm{Xu}$ et al. reported that N, S co-doped carbon derived from Sophora japonica (SJ) was prepared by a two-step heat treatment process for zinc-air battery. ${ }^{39}$ Despite the above progress to date, the hierarchically porous carbon prepared by SJ used as SCs electrodes has been rarely reported.

In this study, $\mathrm{N}, \mathrm{O}$ and $\mathrm{P}$ co-doped honeycomb-like hierarchically porous carbon (N-O-P-HHPC) derived from SJ was firstly prepared by one-step activation and carbonization processes for high performance SCs. The obtained N-O-P-HHPC indicates a higher energy density and long life stability. Therefore, a facile and economic strategy to fabricate N, O and P co-doped HHPC is a considerable candidate for high-performance SCs.

\section{Experimental section}

\section{Synthesis of N-P-HHPC}

The SJ without any impurities was collected from Suzou city in Anhui province and freeze-dried. In brief, a certain mass of $\mathrm{KOH}$ was dissolved in deionized water $(50 \mathrm{~mL})$, and mixed with $6 \mathrm{~g} \mathrm{SJ}$. After soaking for $48 \mathrm{~h}$, the mixed samples were freeze-dried. The mixture samples were heated to $800{ }^{\circ} \mathrm{C}$ for $2 \mathrm{~h}$ at the rate of $2{ }^{\circ} \mathrm{C} \min ^{-1}$. After cooled down to room temperature, the black mixture samples were soaked into $\mathrm{HCl}$ solution $\left(6 \mathrm{~mol} \mathrm{~L}^{-1}, 50\right.$ $\mathrm{mL}$ ) at $65^{\circ} \mathrm{C}$ for $48 \mathrm{~h}$. Then, the obtained materials were washed to $\mathrm{PH}=7$ and dried at $65{ }^{\circ} \mathrm{C}$ for $24 \mathrm{~h}$. In order to distinguish different samples, the as-prepared samples were labeled as $\mathrm{N}-\mathrm{O}-$ P-HHPC-0, N-O-P-HHPC-1, N-O-P-HHPC-3 and N-O-P-HHPC-6, corresponding to $0 \mathrm{~g}, 1 \mathrm{~g}, 3 \mathrm{~g}$ and $6 \mathrm{~g} \mathrm{KOH}$, respectively. The elemental contents of all samples were measured by using the X-ray photoelectron spectroscope (XPS, Thermo Scientific ESCALab 250Xi system). The detailed characterizations and electrochemical measurements are shown in the ESI. $\dagger$

\section{Results and discussion}

The surface microstructures and morphologies of N-O-P-HHPC-0, N-O-P-HHPC-1, N-O-P-HHPC-3 and N-O-P-HHPC-6 were measured by using SEM. Notably, it can be seen that N-O-P-HHPC0 shows smooth block structure without obviously porous structure as shown in Fig. 1a and b. With the increase mass of $\mathrm{KOH}$, the proportion of micropores increases and the pore density also
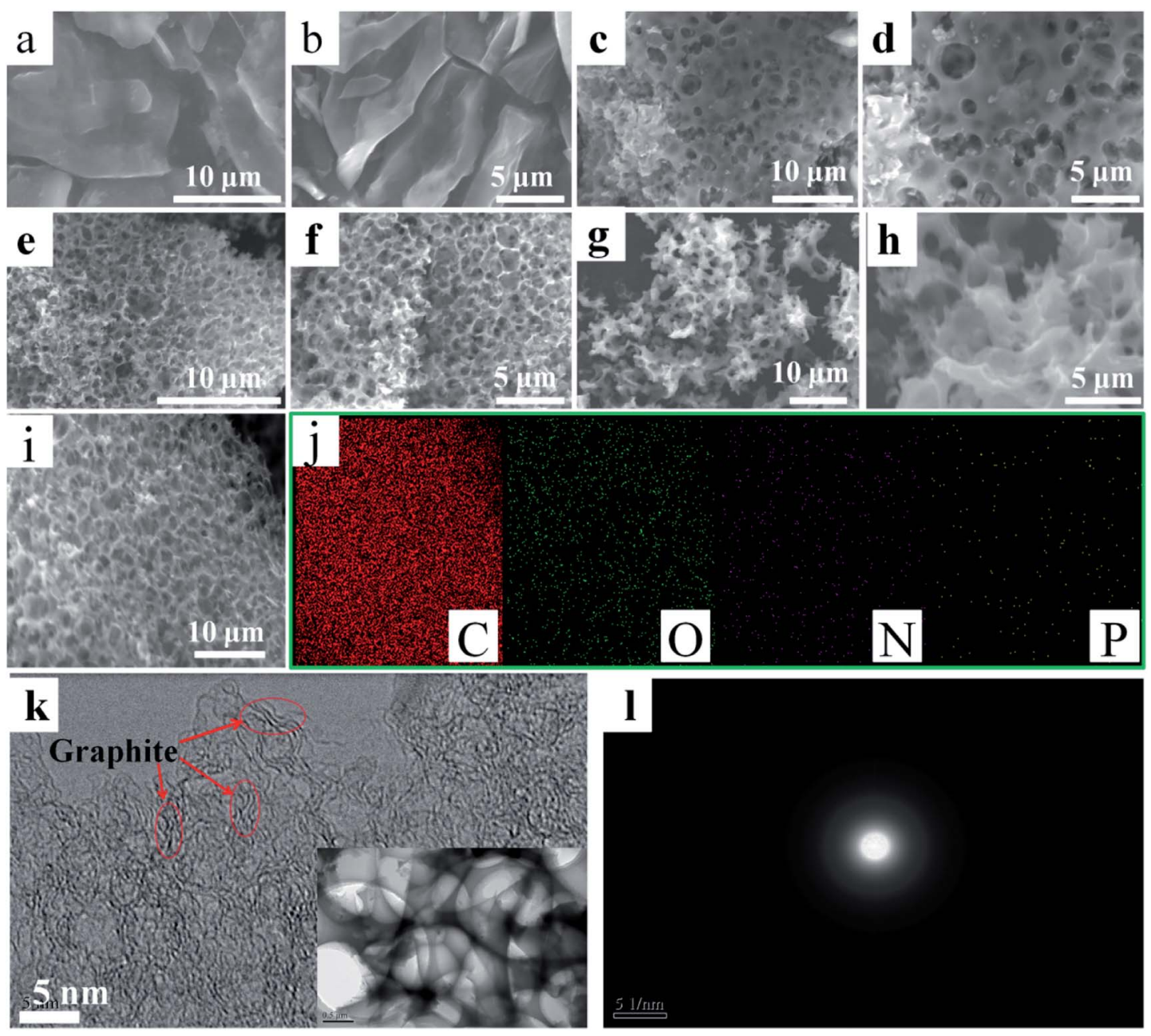

Fig. 1 SEM images of N-O-P-HHPC-O ( $a$ and b), N-O-P-HHPC-1 ( $c$ and d), N-O-P-HHPC-3 (e and f) and N-O-P-HHPC-6 (g and h), SEM image of N-O-P-HHPC-3 (i), and corresponding elemental mapping images of $\mathrm{C}, \mathrm{O}, \mathrm{N}$ and $\mathrm{P}$ (j). HRTEM image (k) and SAED pattern (l) of $\mathrm{N}-\mathrm{O}-\mathrm{P}-$ HHPC-3. Inset of $(k)$ is TEM image of N-O-P-HHPC-3. 
increases. Therefore, appropriate porous structure can be obtained by controlled the mass of KOH. As shown in Fig. 1c and d, N-O-P-HHPC-1 displays obvious porous structure due to the activating agent of $\mathrm{KOH}$. When the mass value of $\mathrm{KOH}$ is $3 \mathrm{~g}$, N-O-PHHPC-3 appears more porous structure, which looks like honeycomb structure as shown in Fig. 1e and f. With the mass of $\mathrm{KOH}$ increases to $6 \mathrm{~g}$, the honeycomb-like porous structure disappears as shown in Fig. $1 \mathrm{~g}$ and $\mathrm{h}$. The SEM image of N-O-P-HHPC-3 and corresponding elemental mapping images of $\mathrm{C}, \mathrm{O}, \mathrm{N}$ and $\mathrm{P}$ are shown in Fig. 1i and $\mathrm{j}$, indicating the uniform $\mathrm{C}, \mathrm{O}, \mathrm{N}$ and $\mathrm{P}$ distributions. The porous framework structure of N-O-P-HHPC-3 was further characterized by TEM, HRTEM and SAED. As shown in Fig. 1k, N-O-P-HHPC-3 exhibits 3D interconnected porous structure, which can facilitate ion electrolyte transport and reduce ion diffusion resistance. It is noted that there are some graphiticlike structure could be observed in HRTEM image, which is attributed to the interlayer spacing of graphite (002). In addition, the diffraction ring in the corresponding SAED pattern is blurred, indicating the defected/disordered structure, which can provide more adsorption sites to enhance the capacitance performance.

To further testify the defected/disordered structure of asprepared samples, XRD patterns and Raman spectra were employed by X-ray diffraction (Rigaku Smartlab) and Raman spectroscopy (LabRAM HR800), and the results are shown in Fig. 2. As-prepared samples exhibit a broad peak at about $23^{\circ}$ and a weak peak at $46^{\circ}$ as shown in Fig. 2a, corresponding to (002) and (101), which reflects the defected/disordered structure. ${ }^{39}$ Compared with N-O-P-HHPC-0, the broad peak at about $23^{\circ}$ of others shift to a lower angle, indicating the defective or disorderly carbon. Moreover, it is clear that the patterns of N-O-P-HHPC-1, N-O-P-HHPC-3 and N-O-P-HHPC-6 at $2 \theta<10^{\circ}$ show an obviously rising tendency, which means the presence of more micropores, ${ }^{21,27}$ resulting in an outstanding specific capacitance. It is consistent with HRTEM results, which favors the improvement of capacitance performance. As shown in Fig. $2 b$, it is obviously found that the peaks of Raman spectra at about $1350 \mathrm{~cm}^{-1}$ and $1580 \mathrm{~cm}^{-1}$ are assigned to D band (defective or disordered carbon) and $\mathrm{G}$ band (graphitic layers), ${ }^{39,40}$ respectively. As known, as-prepared samples with defected/disordered structure can provide more adsorption sites and the channel for ions to rapidly diffuse and transfer, resulting in the enhanced electrochemical performance. The degree of defective or disordered $\left(I_{\mathrm{D}} / I_{\mathrm{G}}\right)$ is presented in Fig. $2 \mathrm{~b}$. The $I_{\mathrm{D}} / I_{\mathrm{G}}$ ratio values of N-O-PHHPC-0, N-O-P-HHPC-1, N-O-P-HHPC-3 and N-O-P-HHPC-6 are $0.90,1.01,1.10$ and 0.99 , respectively. Obviously, the $I_{\mathrm{D}} / I_{\mathrm{G}}$ ratio value of N-O-P-HHPC-3 is the largest, indicating the degree of defect or disorder is the largest. ${ }^{41}$

In order to investigate the pore structure more accurately, $\mathrm{N}_{2}$-adsorption and desorption measurement was characterized by ASAP 2020 system (Micromeritics, Norcross, GA). The $\mathrm{N}_{2}$ adsorption and desorption isotherms are presented in Fig. 2c. Compared with N-O-P-HHPC-0, $\mathrm{N}_{2}$-adsorption and desorption
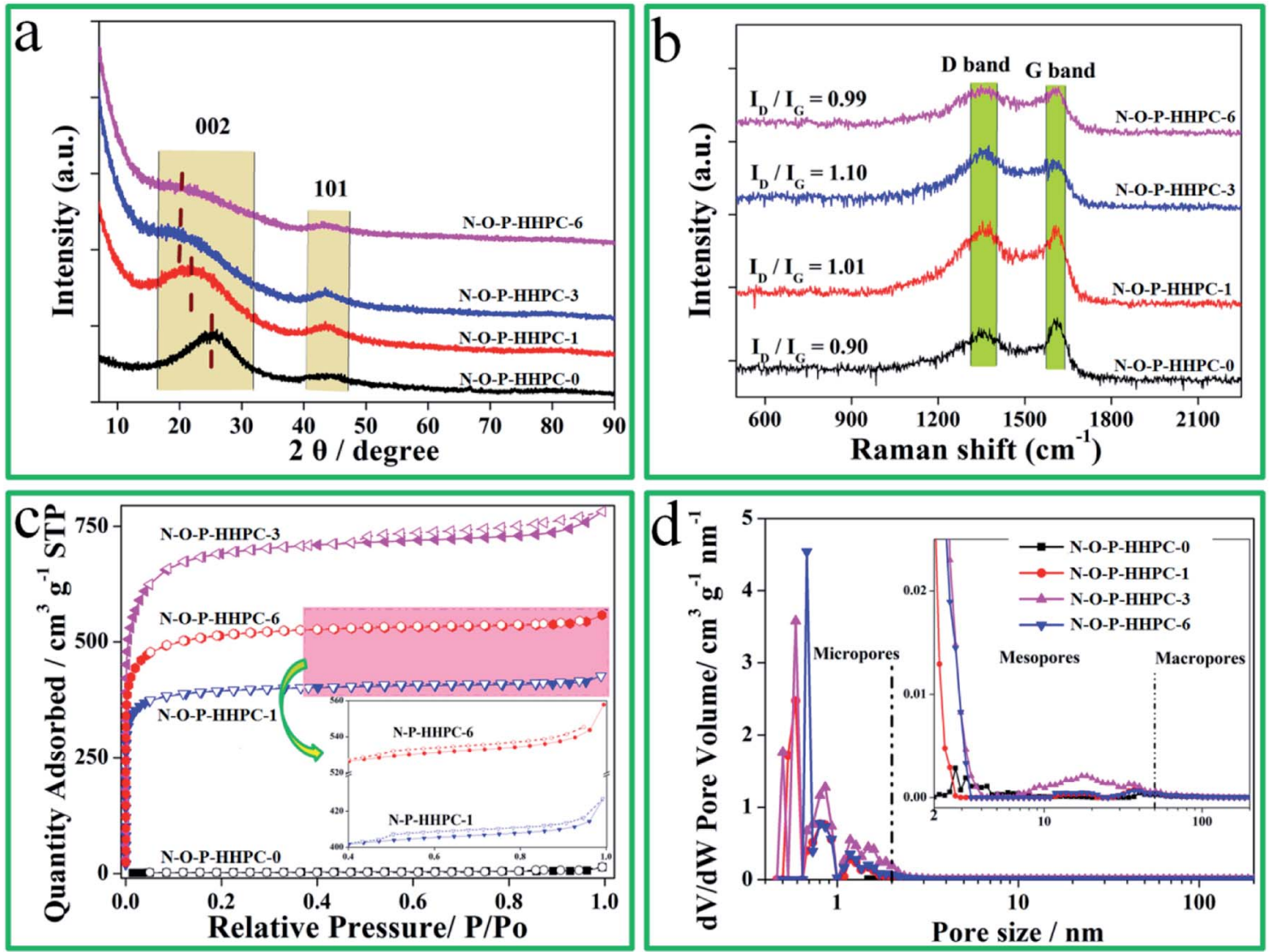

Fig. 2 XRD patterns (a), Raman spectra (b), $\mathrm{N}_{2}$-adsorption and desorption isotherms (c) and pore size distributions (d) of N-O-P-HHPC-0, N-OP-HHPC-1, N-O-P-HHPC-3 and N-O-P-HHPC-6. 
Table 1 SSA ( $S_{\mathrm{BET}}, S_{\text {mic }}$ and $S_{\text {ext }}$ ), $V_{\text {total, }}$ the elemental contents (C, O, N and P) and $R_{c t}$ of N-O-P-HHPC-0, N-O-P-HHPC-1, N-O-P-HHPC-3 and $\mathrm{N}-\mathrm{O}-\mathrm{P}-\mathrm{HHPC}-6$

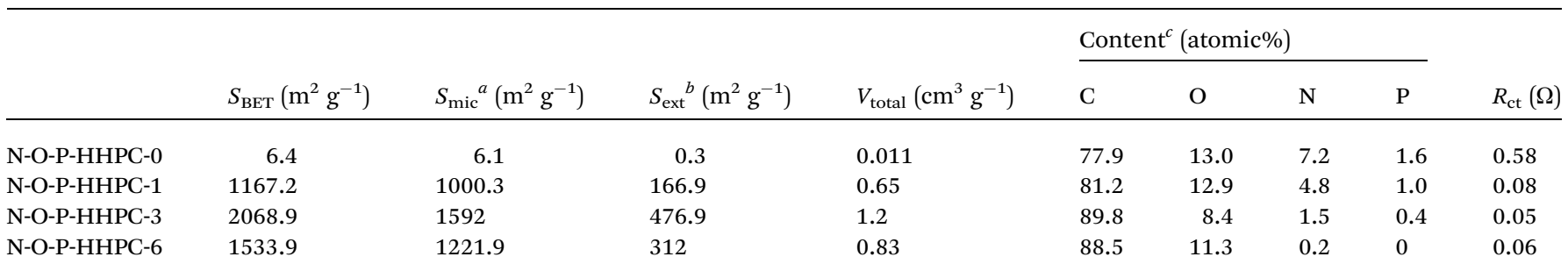

${ }^{a}$ Specific surface area of micropores. ${ }^{b}$ Specific surface area of other pores. ${ }^{c}$ The elemental contents of samples were employed by XPS.

isotherms of others exhibit type-I and type-IV isotherm with a strong adsorption $\left(P / P_{0}<0.05\right)$, broadened knees $\left(0.05<P / P_{0}<\right.$ $0.4)$ and the hysteresis $\left(P / P_{0}>0.5\right)$, indicating the existence of micropores and mesopores. ${ }^{37,42,43}$ The $\mathrm{N}_{2}$ adsorption amount of $\mathrm{N}-\mathrm{O}-\mathrm{P}-\mathrm{HHPC}-3$ is the largest and the hysteresis is more obviously, which means the existence of more micropores and mesopores. The SSA and pore size distributions of N-O-P-HHPC0, N-O-P-HHPC-1, N-O-P-HHPC-3 and N-O-P-HHPC-6 were analyzed via Brunauer-Emmett-Teller model and DFT method from $\mathrm{N}_{2}$-adsorption and desorption isotherms, respectively. The results are shown in Table 1 and Fig. 2d. The N-O-P-HHPC-3 features high surface area $\left(2068.9 \mathrm{~m}^{2} \mathrm{~g}^{-1}\right)$ and the largest pore volume $\left(1.2 \mathrm{~m}^{3} \mathrm{~g}^{-1}\right)$ than those of N-O-P-HHPC-0, N-O-PHHPC-1 and N-O-P-HHPC-6, which indicates that as-prepared N-O-P-HHPC-3 has a superior rate capability.

In order to better prove the element composition of asprepared samples, XPS measurements were characterized by Thermo Scientific ESCALab 250Xi system. As shown in Fig. 3,
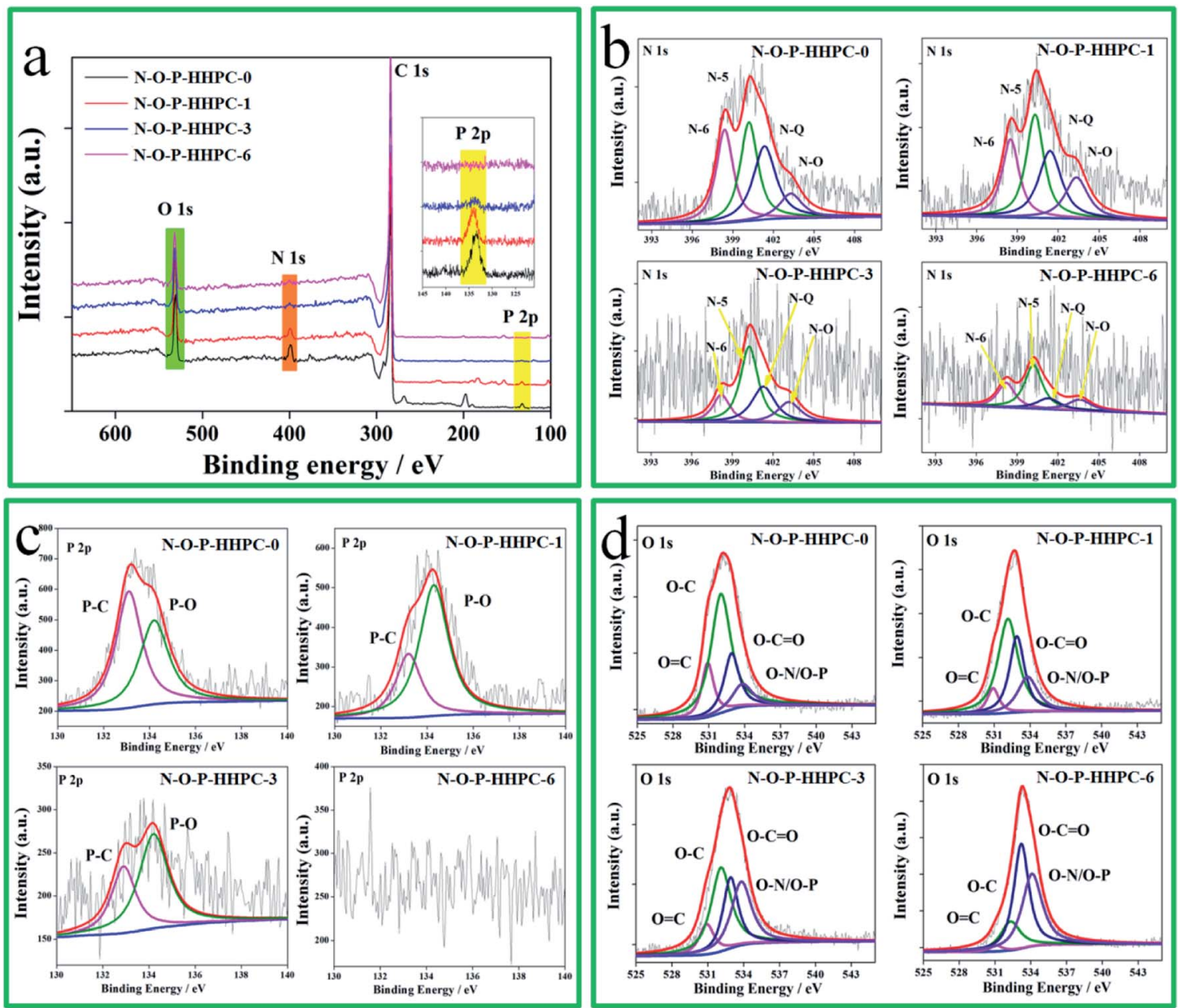

Fig. 3 XPS survey spectra (a), N 1s spectra (b), P 2p spectra (c) and O 1s spectra (d) of N-O-P-HHPC-0, N-O-P-HHPC-1, N-O-P-HHPC-3 and NO-P-HHPC-6. 
there are four mainly peaks at about 533, 400, 284 and $135 \mathrm{eV}$, corresponding to the binding energies of $\mathrm{O} 1 \mathrm{~s}, \mathrm{~N} 1 \mathrm{~s}, \mathrm{C} 1 \mathrm{~s}$ and $\mathrm{P}$ $2 \mathrm{p}$, indicating $\mathrm{N}, \mathrm{O}$ and $\mathrm{P}$ self-doping during carbonizing. ${ }^{17,22,30}$ According to the N, O and P contents of all samples (Table 1), it is obvious found that $\mathrm{N}, \mathrm{O}$ and $\mathrm{P}$ are consumed during carbonization and activation process. The $\mathrm{N}, \mathrm{O}$ and $\mathrm{P}$ contents of N-O-P-HHPC-3 are 1.5, 8.4 and 0.4 atomic\%, respectively. The Fig. $3 \mathrm{~b}-\mathrm{d}$ displays the $\mathrm{N} 1 \mathrm{~s}, \mathrm{P} 2 \mathrm{p}$ and $\mathrm{O} 1 \mathrm{~s}$ of all samples, respectively. The $\mathrm{N}$ 1s of all samples are fitted and shown in Fig. $3 \mathrm{~b}$, which indicates that the $\mathrm{N}$ element mainly includes pyridinic-N-oxide (403.3 eV, N-O) quaternary-N (401.5 eV, N-Q), pyrrolic/pyridone- $\mathrm{N}(400.1 \mathrm{eV}, \mathrm{N}-5)$ and pyridinic-N (398.4 eV, N6). ${ }^{27,30}$ According to previous report, the N-5 and N-6 atoms can mainly contribute pseudocapacitance to enhance the specific capacitance. ${ }^{44}$ Meanwhile, N-Q and N-O can enhance the electron transfer and hydrophilia, ${ }^{33}$ respectively. The $\mathrm{P} 2 \mathrm{p}$ can be fitted into two peaks at $133.2 \mathrm{eV}$ and $134.3 \mathrm{eV}$, corresponding to $\mathrm{P}-\mathrm{C}$ and $\mathrm{P}-\mathrm{O}$, respectively, which can enhance hydrophilia. ${ }^{44,45}$ Obviously, the P element of N-O-P-HHPC-6 is disappeared due to the etching effect of $\mathrm{KOH}$, which improves its contact resistance. ${ }^{30}$ The $\mathrm{O}$ element can be fitted to four peaks at $530.9 \mathrm{eV}$, $532.1 \mathrm{eV} 532.9 \mathrm{eV}$ and $533.8 \mathrm{eV}$, corresponding to $\mathrm{O}=\mathrm{C}, \mathrm{O}-\mathrm{C}$, $\mathrm{O}-\mathrm{C}=\mathrm{O}$ and $\mathrm{O}-\mathrm{N} / \mathrm{O}-\mathrm{P}$, respectively, which can enhance the hydrophilia and some capacitive performance. ${ }^{43,45}$ These results of XPS further confirm that N, O and $\mathrm{P}$ can be self-doped into NO-P-HHPC, resulting in the improved electrochemical performance.

In the three electrode system, the electrochemical performances (CV, EIS and GCD) of N-O-P-HHPC-0, N-O-P-HHPC-1, NO-P-HHPC-3 and N-O-P-HHPC-6 were tested in $6 \mathrm{M} \mathrm{KOH}$, and the results are shown in Fig. 4, S1 and S2. $\dagger$ As shown in Fig. S1, $\dagger$ all CV curves without any redox peak show approximately symmetrical rectangle. The area of CV curve corresponds to the specific capacitance. The larger area of $\mathrm{CV}$ curve at the same scan rate indicates the larger specific capacitance. From the Fig. 4a, CV curve of N-O-P-HHPC-3 displays the largest area than others, indicating the largest specific capacitance. Meanwhile, the GCD curve of N-O-P-HHPC-3 exhibits the longest discharge time, also meaning the largest specific capacitance, which is consistent with the results of CV curves. The largest specific capacitance of N-O-P-HHPC-3 is $386 \mathrm{~F} \mathrm{~g}^{-1}$ at $1 \mathrm{~A} \mathrm{~g}^{-1}$, because the numerous micropores and mesopores provide more adsorption sites and $\mathrm{N}, \mathrm{O}$ and $\mathrm{P}$ co-doping can produce pseudocapacitance. EIS curves of N-O-P-HHPC-0, N-O-P-HHPC-1, NO-P-HHPC-3 and N-O-P-HHPC-6 are displayed in Fig. 4c. It is obviously seen that N-O-P-HHPC-3 exhibits more vertical slope line in the low frequency region, indicating more capacitance performance. In the high frequency region, the charge transfer
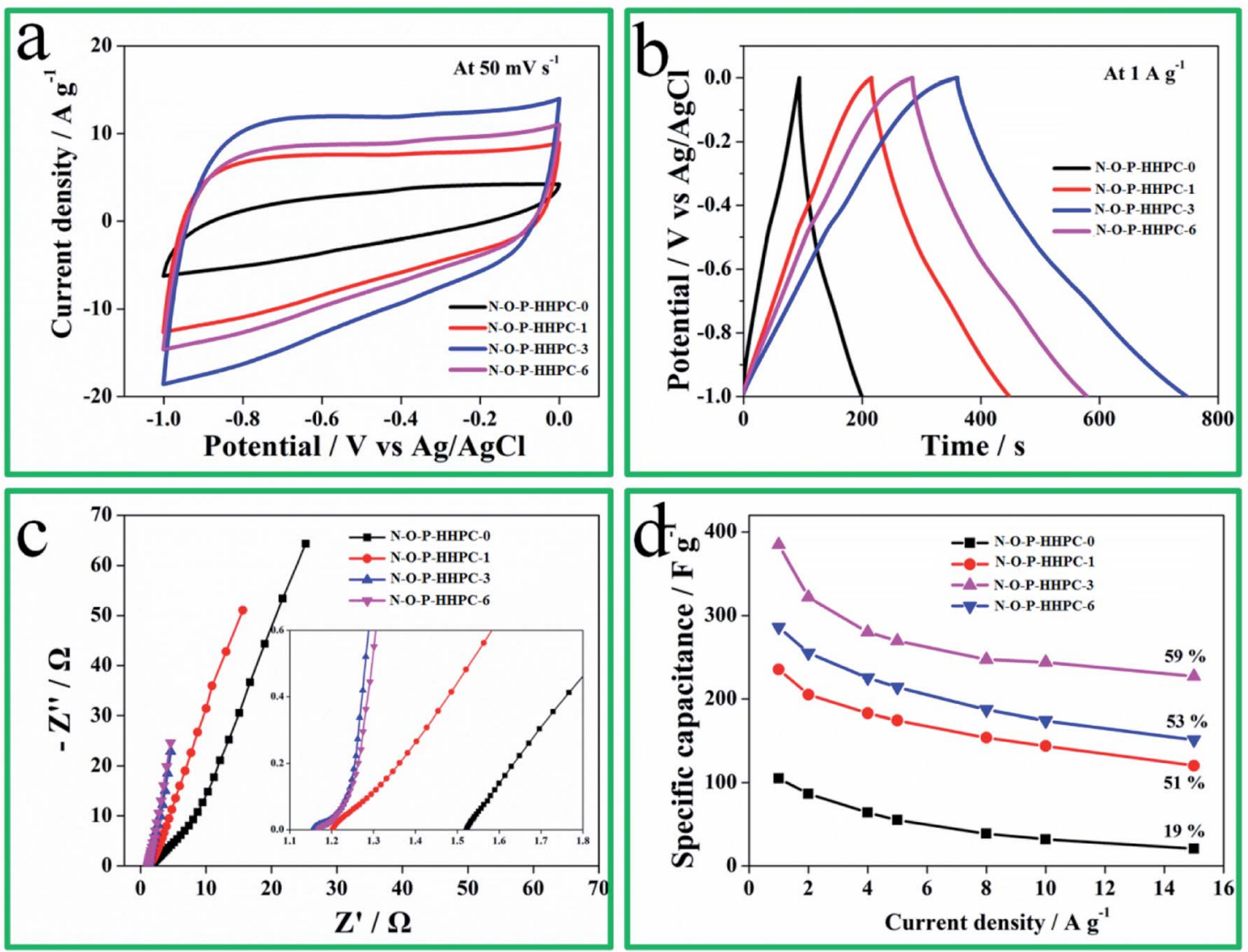

Fig. 4 The electrochemical performances of N-O-P-HHPC-0, N-O-P-HHPC-1, N-O-P-HHPC-3 and N-O-P-HHPC-6 tested in 6 KOH using the three electrode system. (a) CV curves at $50 \mathrm{mV} \mathrm{s}^{-1}$. (b) GCD curves at $1 \mathrm{~A} \mathrm{~g}^{-1}$. (c) EIS curves, the inset at lower right corner is the zoom-in Nyquist plots. (d) The specific capacitances at different current densities. 

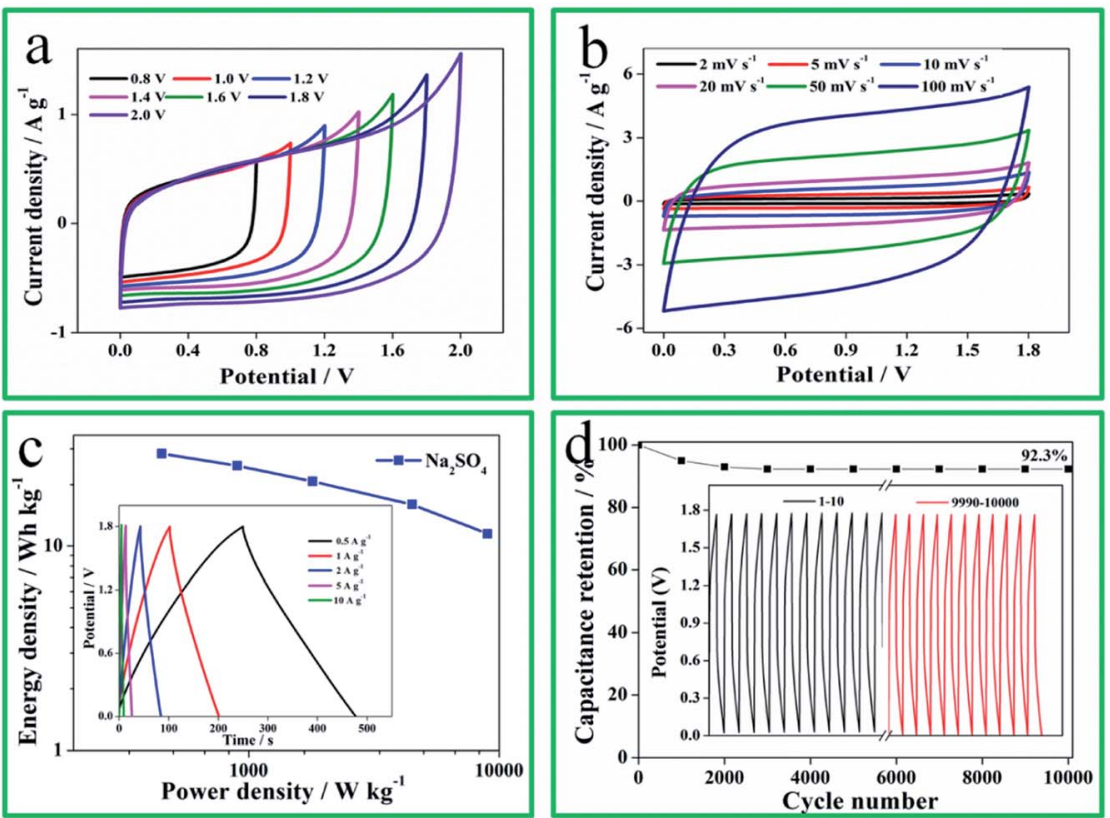

Fig. 5 The electrochemical performances of N-O-P-HHPC-3//N-O-P-HHPC-3 symmetrical SCs using $1 \mathrm{M} \mathrm{Na}_{2} \mathrm{SO}_{4}$ gel electrolyte. (a) CV curves of different potential windows at $10 \mathrm{mV} \mathrm{s}^{-1}$. (b) CV curves of different scan rates. (c) Ragone plot, inset of (c) is GCD curves at different current densities. (d) Cycling stability efficiency at $10 \mathrm{~A} \mathrm{~g}^{-1}$, inset of (d) is the first 10 cycles and the last 10 cycles.

resistance $\left(R_{\mathrm{ct}}=0.05 \Omega\right)$ of N-O-P-HHPC-3 displays the smallest in Table 1, due to its interconnected porous structure. At the same time, the curves of specific capacitance versus current density (as shown in Fig. 4d) for the N-O-P-HHPC-3 also shows the highest specific capacitance. Moreover, the capacitance retention of N-O-P-HHPC-3 is $59 \%$ at $15 \mathrm{~A} \mathrm{~g}^{-1}$, which is higher than N-O-P-HHPC-1 (51\%) and N-O-P-HHPC-6 (53\%), due to its larges specific surface area with 3D hierarchically porous structure (micropores, mesopores and macropores). The existence of numerous micropores can provide ion adsorption sites,
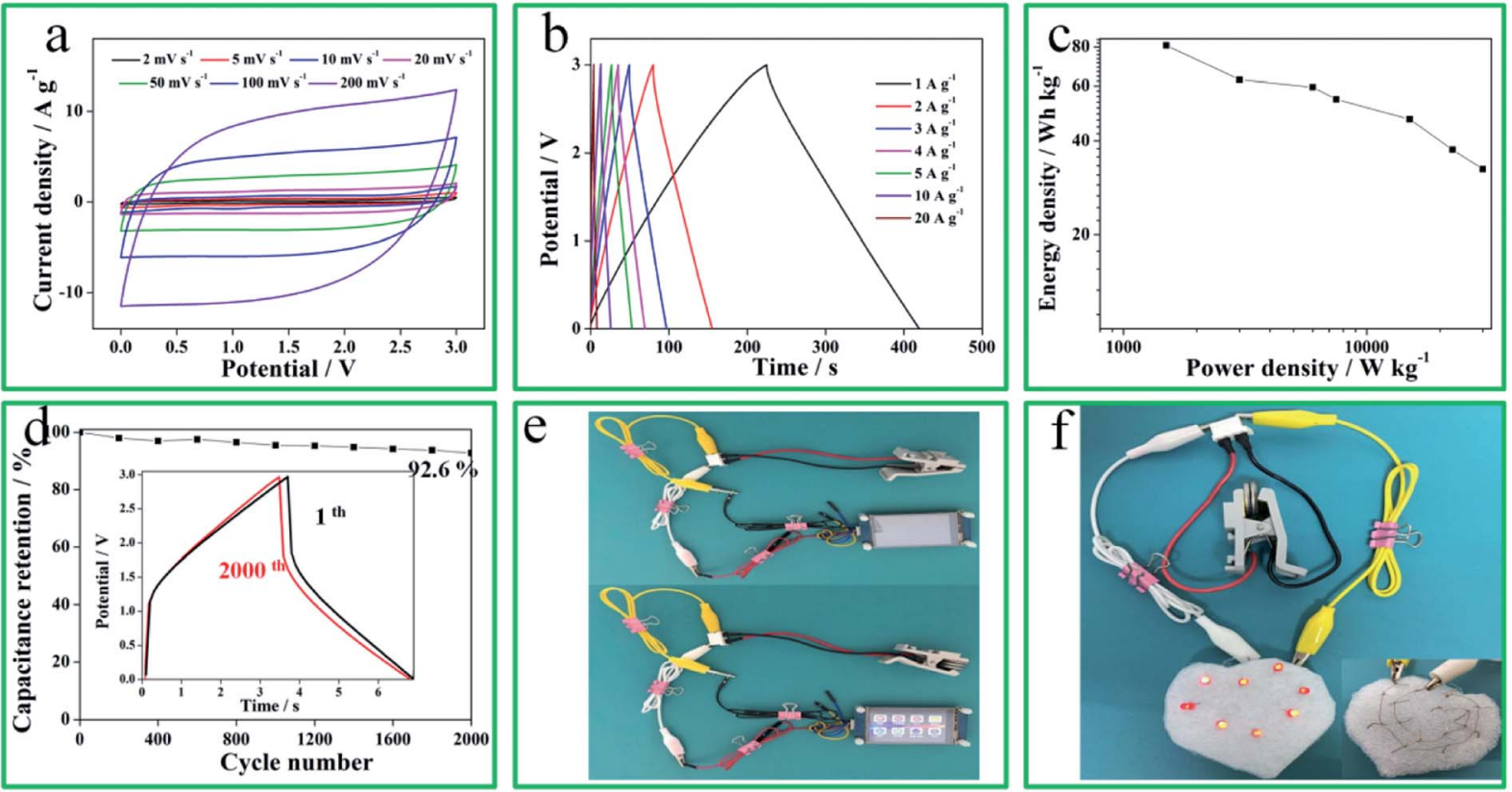

Fig. 6 The electrochemical performances of $\mathrm{N}-\mathrm{O}-\mathrm{P}-\mathrm{HHPC}-3 / / \mathrm{N}-\mathrm{O}-\mathrm{P}-\mathrm{HHPC}-3$ symmetrical SCs using EMIMBF4 as electrolyte. (a) CV curves at $2-200 \mathrm{mV} \mathrm{s}^{-1}$. (b) GCD curves at 1-20 A g ${ }^{-1}$. (c) Ragone plot. (d) The capacitance retention vs. cycle numbers. The inset of GCD curves at 1 st and 2000th. (e) Digital display lighted by three N-O-P-HHPC-3//N-O-P-HHPC-3 symmetrical SCs devices connected in series. (f) The eight red LED bulbs with heart shape powered by two coin-type symmetrical SCs connected in series. 
and the mesoporous and macroporous structures can form ion fast channels, resulting in enhanced electrochemical performances.

To further reveal the capacitance performances, the symmetrical SCs was constructed by N-O-P-HHPC-3 electrode and tested using $1 \mathrm{M} \mathrm{Na}_{2} \mathrm{SO}_{4}$ gel electrolyte by two electrodes system, and the results are shown in Fig. 5. Notable, the potential window of the N-O-P-HHPC-3 |N-O-P-HHPC-3 symmetrical SCs can reach up to $1.8 \mathrm{~V}$ (Fig. 5a). The CV curves of the N-O-P-HHPC-3\|N-O-P-HHPC-3 symmetrical SCs at different scan rates $\left(2-100 \mathrm{mV} \mathrm{s}^{-1}\right)$ display approximately symmetric rectangle in Fig. 5b, owning to its hierarchically porous structure that provides more adsorption sites and the channel for ions to rapidly diffuse and transfer. Meanwhile, GCD curves at $0.5 \mathrm{~A} \mathrm{~g}^{-1}$ show equicrural triangle with a small internal resistance (IR) drop. The Ragone plot of the N-O-PHHPC-3//N-O-P-HHPC-3 symmetrical SCs is shown in Fig. 5c. When the power density is $449.9 \mathrm{~W} \mathrm{~kg}^{-1}$, the energy density can be reach up to $28.4 \mathrm{~W} \mathrm{~h} \mathrm{~kg}^{-1}$, which is higher than the reported energy density of biomass carbon based supercapacitors (Table S1 $\dagger$ ). When the power density increases to $8992.2 \mathrm{~W} \mathrm{~kg}^{-1}$, the energy density of $11.5 \mathrm{~W} \mathrm{~h} \mathrm{~kg}^{-1}$ can still be delivered. Meanwhile, the cycling stability of the symmetrical SCs with the potential window of $1.8 \mathrm{~V}$ is analyzed at $10 \mathrm{~A} \mathrm{~g}^{-1}$ for 10000 cycles, exhibiting an outstanding long life cycle (capacitance retention of 92.3\%) in Fig. 5d.

To further investigate the superior capacitive performances of N-O-P-HHPC-3, a two-electrode coin-type symmetrical SCs was assembled with EMIMBF4 as electrolyte. As shown in Fig. 6a and b, CV curves display rectangular shapes and GCD plots exhibit perfect linear and symmetrical shapes, indicating ideal EDLC behavior. Meanwhile, the potential window of the coin-type symmetrical SCs can reach to $3 \mathrm{~V}$, which is better to enhance the energy density. The Ragone plot of the coin-type symmetrical SCs is shown in Fig. 6c. When the power density increases, the energy density decreases. The ultrahigh energy density is $80.8 \mathrm{~W} \mathrm{~h} \mathrm{~kg}^{-1}$ at $1500 \mathrm{~W} \mathrm{~kg}^{-1}$, which is larger than previous literature reports (Table S1†). The cycling stability of the coin-type symmetrical SCs was analyzed, and the results are shown in Fig. 6d. The two-electrode coin-type symmetrical SCs demonstrates an excellent long life cycles. The capacitance retention reaches up to $92.6 \%$ after 2000 cycles (GCD curves) at $20 \mathrm{~A} \mathrm{~g}^{-1}$. Benefiting from the integrated high energy and power properties, two coin-type symmetrical SCs in series can power a small display screen (5 V, Fig. 6e) and eight red LED modules (Fig. 6f), indicating its great potential for high-performance SCs application.

\section{Conclusion}

In summary, the $\mathrm{N}, \mathrm{O}$ and $\mathrm{P}$ co-doped honeycomb-like hierarchically porous carbon was successfully prepared from Sophora japonica by a facile, relatively green and efficient strategy. The specific capacitance of the optimized N-O-P-HHPC-3 can reaches up to $386 \mathrm{~F} \mathrm{~g}^{-1}$ at current density of $1 \mathrm{~A} \mathrm{~g}^{-1}$ with the capacitance retention of $59 \%$. The excellent performance of $\mathrm{N}$ O-P-HHPC-3 is assigned to the contribution of the largest specific surface area of $2068.9 \mathrm{~m}^{2} \mathrm{~g}^{-1}$ with interconnect honeycomb-like hierarchical porous structure (micropores, mesopores and macropores) and $\mathrm{N}$ (1.5 atomic\%), O (8.4 atomic\%) P (0.4 atomic\%) co-doping. Meanwhile, the $1.8 \mathrm{~V} \mathrm{N-O-}$ P-HHPC-3//N-O-P-HHPC-3 symmetrical SCs using high-voltage $1 \mathrm{M} \mathrm{Na}_{2} \mathrm{SO}_{4}$ gel electrolyte shows an outstanding cycling stability (7.7\% capacitance loss after 10000 charge and discharge cycles). Furthermore, the two-electrode coin-type symmetrical SCs exhibits an ultrahigh the energy density of $80.8 \mathrm{~W} \mathrm{~h} \mathrm{~kg}^{-1}$ at $1500 \mathrm{~W} \mathrm{~kg}^{-1}$ using EMIMBF4 as electrolyte. When the two coin-type symmetrical SCs are connected in series, the eight red LED in parallels and the small display screen can be powered, which directly demonstrates tremendous opportunities to be applied in high performance energy storage.

\section{Conflicts of interest}

There are no conflicts to declare.

\section{Acknowledgements}

This work is financially supported by the Provincial Natural Science Foundation of Anhui (1908085ME120), Primary Research and Development Program of Anhui Province (201904a05020087), and the Scientific Research Foundation of Suzhou University (2016XJXS05 and 2018SZXYDZXZ02), Outstanding Youth Talents in Anhui Provincial Education Department (2017GXBJZD47), Innovative Research Team of Anhui Provincial Education Department (2016SCXPTTD), and Key Discipline of Material Science and Engineering of Suzhou University (2017XJZDXK3).

\section{References}

1 J. Yan, Q. Wang, T. Wei and Z. Fan, Adv. Energy Mater., 2014, 4, 1300816.

2 Y. Liu, B. Huang, X. Lin and Z. Xie, J. Mater. Chem. A, 2017, 5, 13009-13018.

3 J. Sun, S. Yang, C. Yang, Q. Jia, X. Yang and B. Cao, New J. Chem., 2019, 43, 10103-10108.

4 Z. Gao, C. Bumgardner, N. Song, Y. Zhang, J. Li and X. Li, Nat. Commun., 2016, 7, 11586.

5 B. Li, F. Dai, Q. Xiao, L. Yang, J. Shen and C. Zhang, Energy Environ. Sci., 2016, 9, 102-106.

6 Y. Li, D. Zhang, J. He, Y. Wang, X. Zhang and Y. Zhang, Sustainable Energy Fuels, 2019, 3, 499-507.

7 W. Qin, T. Chen, L. Pan, L. Niu, B. Hu and D. Li, Electrochim. Acta, 2015, 153, 55-61.

8 L. Lai, Y. Zhao, S. Ying, L. Li, Z. Ma and L. Pan, RSC Adv., 2018, 8, 18714-18722.

9 X. T. Xu, Y. Liu, M. Wang, C. Zhu, T. Lu and R. Zhao, Electrochim. Acta, 2016, 193, 88-95.

10 Y. J. Li, G. Zhu, H. L. Huang, M. Xu, T. Lu and L. K. Pan, J. Mater. Chem. A, 2019, 7, 9040-9050.

11 H. Jin, J. Li, Y. Yuan, J. Wang, J. Lu and S. Wang, Adv. Energy Mater., 2018, 8, 1801007. 
12 G. Zhao, Y. Li, G. Zhu, J. Shi, T. Lu and L. Pan, Ionics, 2019, 25, 3935-3944.

13 J. Azadmanjiri, V. K. Srivastava, P. Kumar, M. Nikzad, J. Wang and A. Yu, J. Mater. Chem. A, 2018, 6, 702-734.

14 A. Borenstein, O. Hanna, R. Attias, S. Luski, T. Brousse and D. Aurbach, J. Mater. Chem. A, 2017, 5, 12653-12672.

15 K. Jayaramulu, D. P. Dubal, B. Nagar, V. Ranc, O. Tomanec and M. Petr, Adv. Mater., 2018, 30, 1705789.

16 Y. Hao, F. Xu, M. Qian, J. Xu, W. Zhao and F. Huang, RSC Adv., 2017, 7, 10901-10905.

17 G. Ren, Y. Li, Q. Chen, Y. Qian, J. Zheng and Y. Zhu, ACS Sustainable Chem. Eng., 2018, 6, 16032-16038.

18 H. Niu, B. Luo, N. Xin, Y. Liu and W. Shi, J. Alloys Compd., 2019, 785, 374-381.

19 J. Huo, P. Zheng, X. Wang and S. Guo, Appl. Surf. Sci., 2018, 442, 575-580.

20 Q. Wang, H. Liang and D. Wu, RSC Adv., 2019, 9, 1752117529.

21 G. Zhu, G. Zhao, J. Shi and W. Ou-Yang, RSC Adv., 2019, 9, 17308-17317.

22 G. Zhao, Y. Li, G. Zhu, J. Shi, T. Lu and L. Pan, ACS Sustainable Chem. Eng., 2019, 7, 12052-12060.

23 H. Cheng, X. Zhou, A. Gao, F. Yi, D. Shu and X. Song, Electrochim. Acta, 2018, 292, 20-30.

24 N. Wang, C. Wang, L. He, Y. Wang, W. Hu and S. Komarneni, Electrochim. Acta, 2019, 298, 717-725.

25 Y. Wang, M. Zhang, D. Pan, Y. Li, T. Ma and J. Xie, Electrochim. Acta, 2018, 266, 242-253.

26 Z. Zhao and Y. Xie, J. Power Sources, 2018, 400, 264-276.

27 G. Zhao, C. Chen, D. Yu, L. Sun, C. Yang and H. Zhang, Nano Energy, 2018, 47, 547-555.

28 L. Guan, L. Pan, T. Peng, C. Gao, W. Zhao, Z. Yang, H. Hu and M. Wu, ACS Sustainable Chem. Eng., 2019, 7, 8405-8412.
29 X. Chen, M. Chi, L. Xing, X. Xie, S. Liu, Y. Liang, M. Zheng, H. Hu, H. Dong, Y. Liu, S. Ping and Y. Xiao, ACS Sustainable Chem. Eng., 2019, 7, 5845-5855.

30 T. X. Shang, X. X. Cai and X. J. Jin, $R S C A d v .$, 2015, 5, 1643316438.

31 V. Sattayarut, T. Wanchaem, P. Ukkakimapan, V. Yordsri, P. Dulyaseree and M. Phonyiem, RSC Adv., 2019, 9, 2172421732.

32 D. Wu, J. Cheng, T. Wang, P. Liu, L. Yang and D. Jia, ACS Sustainable Chem. Eng., 2019, 7, 12138-12147.

33 G. Lin, R. Ma, Y. Zhou, Q. Liu, X. Dong and J. Wang, Electrochim. Acta, 2018, 261, 49-57.

34 B. Liu, Y. Liu, H. Chen, M. Yang and H. Li, J. Power Sources, 2017, 341, 309-317.

35 W. Zhang, N. Lin, D. Liu, J. Xu, J. Sha and J. Yin, Energy, 2017, 128, 618-625.

36 Y. Zhou, J. Ren, L. Xia, Q. Zheng, J. Liao and E. Long, Electrochim. Acta, 2018, 284, 336-345.

37 C. Long, X. Chen, L. Jiang, L. Zhi and Z. Fan, Nano Energy, 2015, 12, 141-151.

38 Y. Lu, S. Zhang, J. Yin, C. Bai, J. Zhang and Y. Li, Carbon, 2017, 124, 64-71.

39 X. Li and H. Xu, ChemistrySelect, 2018, 3, 10624-10629.

$40 \mathrm{~J}$. Li, G. Zan and Q. Wu, RSC Adv., 2016, 6, 57464-57472.

41 Z. Pei, H. Li, Y. Huang, Q. Xue, Y. Huang, M. Zhu, Z. Wang and C. Zhi, Energy Environ. Sci., 2017, 10, 742-749.

42 Y. Feng and J. Yao, Ind. Eng. Chem. Res., 2018, 57, 7322-7330.

43 J. Niu, J. Liang, R. Shao, M. Liu, M. Dou and Z. Li, Nano Energy, 2017, 41, 285-292.

44 L. Wan, W. Wei, M. Xie, Y. Zhang, X. Li and R. Xiao, Electrochim. Acta, 2019, 311, 72-82.

45 B. Liu, M. Yang, D. Yang, H. Chen and H. Li, Electrochim. Acta, 2018, 272, 88-96. 